\title{
Comparison of two-point (AutoAnalyzer II) with kinetic methods for transaminase assay
}

\author{
A. F. SMITH AND R. H. TAYLOR \\ From the University Department of Clinical Chemistry, The Royal Infirmary, Edinburgh
}

SYNOPSIS The general performance of the Technicon AutoAnalyzer II method for the routine estimation of serum aspartate and alanine transaminase has been assessed and compared with that of the LKB reaction rate analyser. The results of the two methods correlate, there is little difference in precision, and running costs are comparable. Some of the relative advantages of the two types of analysis are discussed.

Transaminase assays comprise a large proportion of the routine enzyme work load of many laboratories, so that some form of automated analysis is essential in larger clinical chemistry laboratories. At present, the most widely employed methods of analysis are discrete reaction rate analysis or continuous flow analysis.

Reaction rate analysis has several practical and theoretical advantages over methods based on twopoint analysis, such as the ability to measure initial reaction rates, or to select a linear portion of the reaction progress curve in the case of reactions with a 'lag' phase; separate 'blank' determinations are not usually necessary and a wide range of enzyme activity may be measured without dilution of the sample.

However, in some circumstances continuous flow methods may have practical advantages. The equipment is versatile, reliable, and familiar to most laboratory workers, and sample preparation is reduced to a minimum. Furthermore, different analyses may conveniently be grouped together either on a functional basis, eg, 'liver function tests', or for screening or profiling purposes.

The present report is an assessment of the current methods of aspartate transaminase (AST) and alanine transaminase (ALT) activity determination using the Technicon AutoAnalyzer II $^{1}$. The results are compared with those obtained using a reaction rate analyser.

\footnotetext{
${ }^{1}$ Technicon Instruments Co Ltd, Basingstoke, England. Received for publication 15 September 1972.
}

\section{Methods}

CONTINUOUS FLOW EQUIPMENT

Standard Technicon AutoAnalyzer II systems for AST and ALT analysis were tested-Technicon methodology AA II-10 for AST, AA II-22 for ALT (the Technicon SMA 12/60 AutoAnalyzer uses similar methods). An analytical rate of 60 samples per hour (9:1 sample: wash ratio) was used; under these conditions a sample of approximately $0.4 \mathrm{ml}$ is aspirated for both AST and ALT.

Similar reaction principles are used for both AST and ALT analysis. For AST analysis (Fig. 1) serum and substrate are mixed and allowed to react at $37^{\circ}$; the oxaloacetate formed is subsequently allowed to dialyze into a recipient stream containing NADH and malate dehydrogenase. The decrease in absorbance at $340 \mathrm{~nm}$, due to oxidation of NADH to NAD, is measured. The principle of ALT analysis is similar, except that the different substrate is used and the recipient stream entering the dialyzer contains lactate dehydrogenase in place of malate dehydrogenase. In addition, a blank channel, identical to the test channel except that phosphate buffer is used in place of substrate, is necessary in order to correct for pyruvate present in serum.

A commercial quality control serum (Dade, EnzaTrol), the activity of which had been determined previously using a manual spectrophotometric technique (Henry, Chiamori, Golub, and Berkman, 1960), was used for single-point standardization of the instrument. A pooled ox serum of low transaminase activity (10 iu/l) was placed in cups $9,19 \ldots$. 


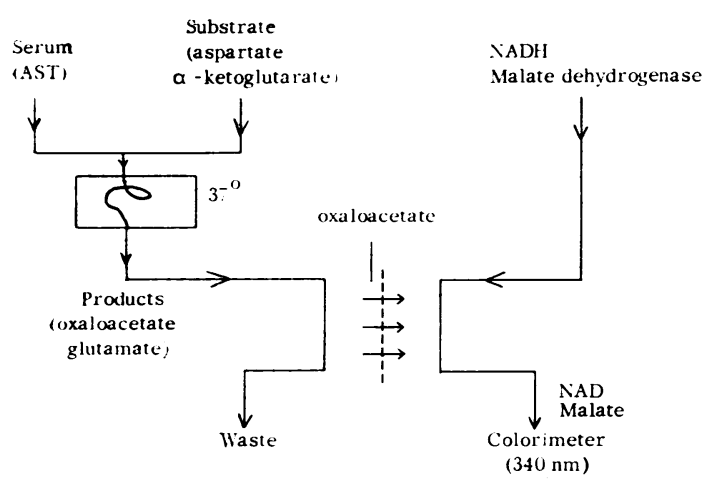

Fig. 1 Aspartate transaminase-principles of the method.

to assess 'baseline drift'. A commercial control serum (Hyland, Special Clinical Chemistry Control Serum) was diluted to obtain an activity of $100 \mathrm{iu} / 1$ and placed in cups $10,20 \ldots$ to assess any withinbatch alterations in the sensitivity of the method. Specimens of SMA 12/60 reference serum (Technicon Instruments Co Ltd) were also included in each analytical run. No alterations in the instrumental 'baseline' or 'sensitivity' settings were made during the analysis, but all transaminase results were subsequently corrected for 'baseline drift' using the results obtained for the pooled ox serum.

\section{REACTION RATE ANALYSIS}

The LKB 8600 reaction rate analyser(LKB-Produkter AB, Bromma, Sweden) was used for kinetic enzyme reaction rate determinations. The reagent concentrations for both AST and ALT were those of Henry, Chiamori, Golub, and Berkman (1960); the performance of the methods has been described previously (Smith, Brown, and Taylor, 1970).

\section{REAGENTS}

'Packaged' reagents for AST and ALT, supplied by Technicon, were used in some of the assessments of the performance of the AutoAnalyzer. Otherwise the reagents were prepared fresh on the day of analysis using the reagent concentrations specified by the manufacturer (Technicon methodology AA II-10 for AST, AA II-22 for ALT).

\section{Results}

GENERAL

No difficulties were experienced in setting up and running the AST analysis The main practical problem lay in the correction of occasional blockages which occurred in the sample probe.
However, ALT analysis initially presented problems, all related to the presence of the blank channel (qv). After the faults due to improper blank subtraction had been diagnosed and corrected, few difficulties with the operation of the method were encountered.

\section{ACCURACY}

There are special problems associated with any assessment of the accuracy of enzyme methods. First, the reaction should be specific for the enzyme being measured, and the observed reaction velocity, or peak height, should be directly proportional to the concentration of enzyme. Secondly, there are problems of standardization; these present difficulties with all methods of enzyme assay, but additional difficulties are encountered when continuous flow analysis is considered. In this section different aspects of the accuracy of transaminase assays are considered under separate headings.

\section{Blank correction}

The AST method has no blank channel and is, therefore, liable to be inaccurate if the serum contains either $(a)$ oxaloacetate or other chemicals which react with NADH and malate dehydrogenase; such materials will cause an apparent increase in enzyme activity; (b) dialyzable substances which absorb at $340 \mathrm{~nm}$; these substances will cause an apparent decrease in enzyme activity.

Specimens submitted to the laboratory for AST analysis were screened for either type of material by running a 'blank' AST analysis, ie, replacing substrate with phosphate buffer. No significant blank effect was noted. However, some laboratories have informed Technicon of difficulties with AST analysis in patients in renal dialysis units. We have confirmed that sera from patients on renal dialysis or with chronic renal failure contain a substance which absorbs at $340 \mathrm{~nm}$ and which may cause inappropriately low or even negative AST results on the AutoAnalyzer. This substance is partially removed from the serum by haemodialysis. In none of the $\mathbf{4 0}$ sera from patients on renal dialysis or with chronic renal disease was the effect sufficient to lower the apparent AST activity by more than $15 \mathrm{iu} / \mathrm{l}$.

The results at first obtained for ALT were too high and suggested that the blank was being only partially subtracted. To test this hypothesis, substrate was pumped through 'blank' and 'test' channels and samples were then aspirated in the normal way: alterations in absorbance in both channels should parallel one another and no net activity should be recorded if the blank and test channels are properly balanced. In practice this was not the case, the poor matching of test and blank 


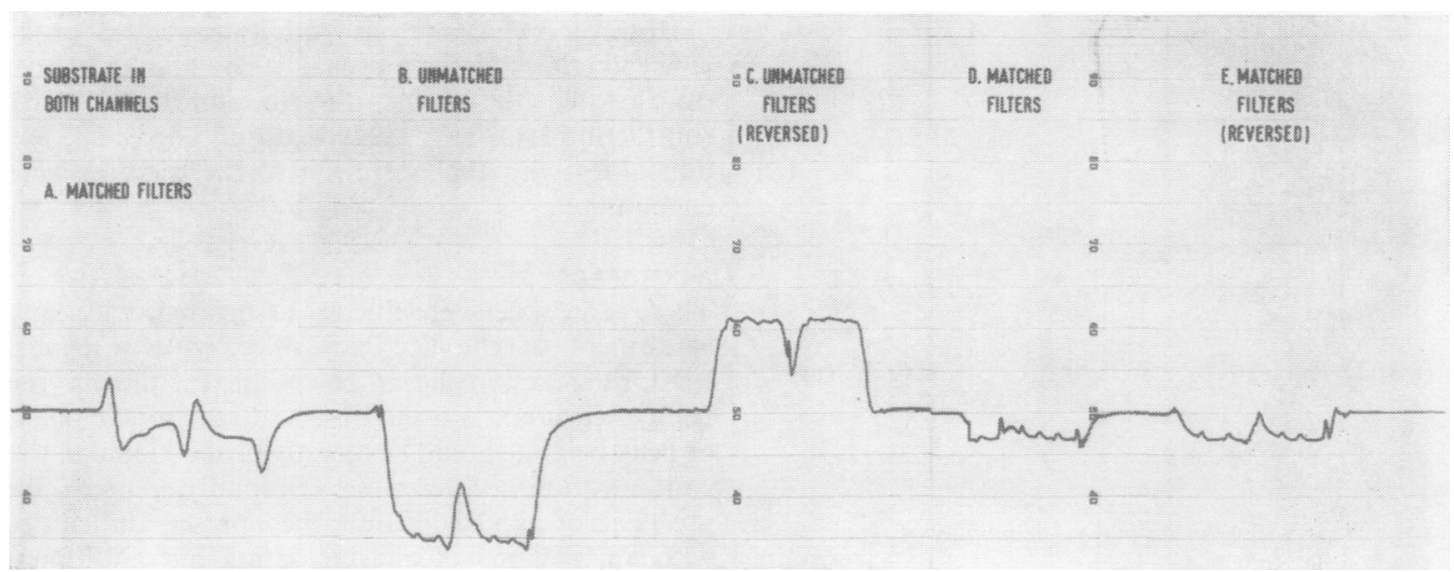

Fig. 2 AutoAnalyzer trace-ALT analysis with substrate pumped through 'test' and 'blank' channels and two identical samples being aspirated. (A) 'Test' and 'blank' $340 \mathrm{~nm}$ filters matched, flow systems slightly out of phase.

(B) Unmatched, wide waveband filter placed in 'blank' position. (C) Filters in (B) reversed. (D) 'Test' and 'blank' filters matched. (E) Filters in (D) reversed. Residual small difference in baseline in (D) and (E) is due to differences in the flow systems.

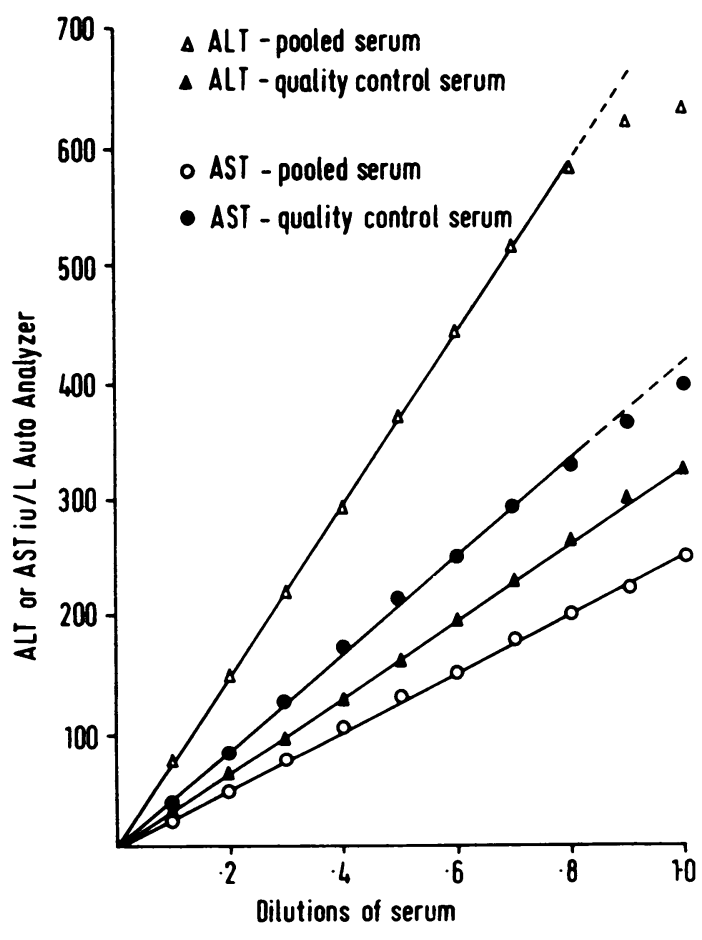

Fig. 3 Effect of serum dilution on enzyme activity. A pooled human serum and a commercial control serum of high transaminase activity were diluted with water to give final enzyme concentrations $0 \cdot 1,0 \cdot 2,0 \cdot 3 \ldots$ of the original serum. The transaminase activity of each dilution was determined on the AutoAnalyzer. channels was found to be due to two factors: (a) improperly matched $340 \mathrm{~nm}$ interference filtersthe major cause of the discrepant results (Fig. 2); (b) difficulties in matching the flow rates in the 'blank' and 'test' pump tubes. After correction of these faults, the blank correcting procedure appeared to be satisfactory.

\section{Linearity}

The relationship between enzyme concentration and enzyme activity (peak height) measured on the AutoAnalyzer was investigated by assaying dilutions of both pooled human serum and commercial control sera (Fig. 3). Good linearity was obtained for the AST method up to $300 \mathrm{iu} / \mathrm{l}$ and for the ALT method up to $600 \mathrm{iu} / 1$.

Correlation with reaction rate analysis

Serum samples were analysed by both the AutoAnalyzer and the reaction rate analyser, both analyses being performed on the same day. Typical examples of a single day's results are shown in Figs. 4 and 5; similar results were obtained on other days (Table I). It can be seen that the results obtained by the two methods are in good agreement on any one day; in particular, if differences due to standardization are allowed for, results in the borderline normal/ abnormal range (30-40 iu/l) rarely differ by more than $3 \mathrm{iu} / \mathrm{l}$-within the limits of precision of the two instruments.

\section{Standardization}

There are two features of the slope of the line relating enzyme activity of serum specimens on the 


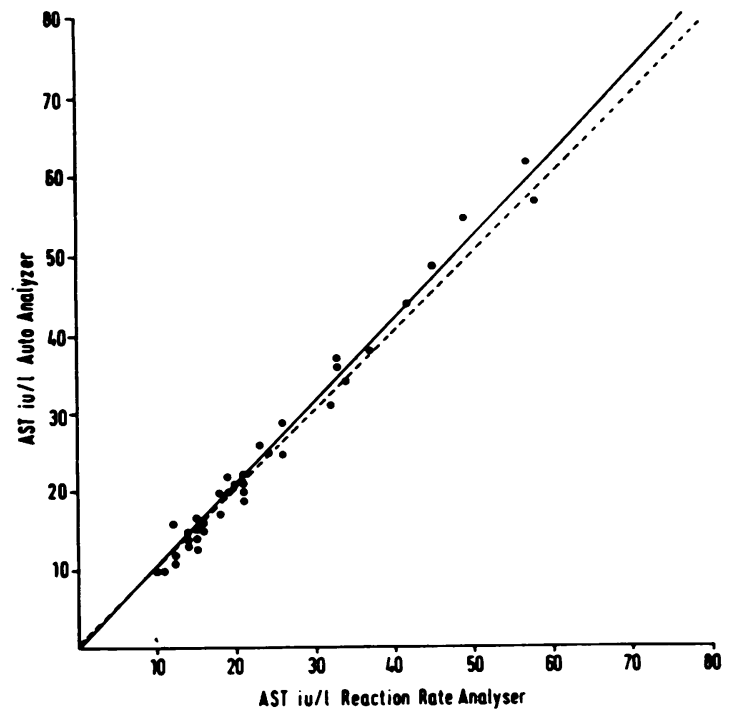

Fig. 4 Correlation between reaction rate analyser and AutoAnalyzer results for patients' samples-AST.

AutoAnalyzer and on the reaction rate analyser (Table I). First, the slope of the line was rather variable: it was possible to attribute this to two factors: (a) within-batch variation in the enzyme activity of the control serum used to standardize the AutoAnalyzer; (b) a period during which the instrument used for preparing dilutions for the

\begin{tabular}{llllll}
\hline Enzyme & Day & $\begin{array}{l}\text { No. of } \\
\text { Specimens }\end{array}$ & $a$ & $b$ & $r$ \\
\hline & 1 & 59 & 0.97 & -2.1 & 0.997 \\
& 2 & 49 & 1.05 & -2.0 & 0.998 \\
AST & 3 & 97 & 1.02 & 1.5 & 0.997 \\
& 4 & 43 & 1.00 & -0.6 & 0.998 \\
& 5 & 45 & 1.02 & -2.0 & 0.998 \\
& 6 & 42 & 1.06 & 0.5 & 0.998 \\
& 7 & 47 & 1.04 & -0.2 & 0.998 \\
& 8 & 37 & 1.04 & -0.1 & 0.998 \\
& 1 & 50 & 1.19 & -10.0 & $0.930^{2}$ \\
& 2 & 46 & 1.13 & -5.4 & $0.990^{3}$ \\
& 3 & 44 & 1.25 & -1.7 & $0.988^{3}$ \\
& 4 & 28 & 1.29 & -0.3 & 0.997 \\
& 5 & 42 & 1.34 & -1.2 & 0.999 \\
& 6 & 46 & $1.10^{4}$ & -0.8 & 0.998 \\
& 7 & 66 & $1.12^{4}$ & -1.7 & 0.998 \\
& 8 & 87 & 1.164 & -1.4 & 0.997 \\
& 9 & 60 & $1.08^{4}$ & -2.2 & 0.998 \\
& 10 & 31 & 1.24 & -0.9 & 0.996 \\
& 11 & 73 & 1.28 & -1.9 & 0.997
\end{tabular}

Table I Correlation between AutoAnalyzer and reaction rate analyser results

${ }^{1}$ Correlation, on separate days, between results on AutoAnalyzer $(y)$ and reaction rate analyser $(x) ; y=a x+b ; r=$ correlation coefficient 'Interference filters not matched

${ }^{3}$ Manifold pump tubing not matched-filters matched

Miscalibration of diluter for Reaction Rate Analyser

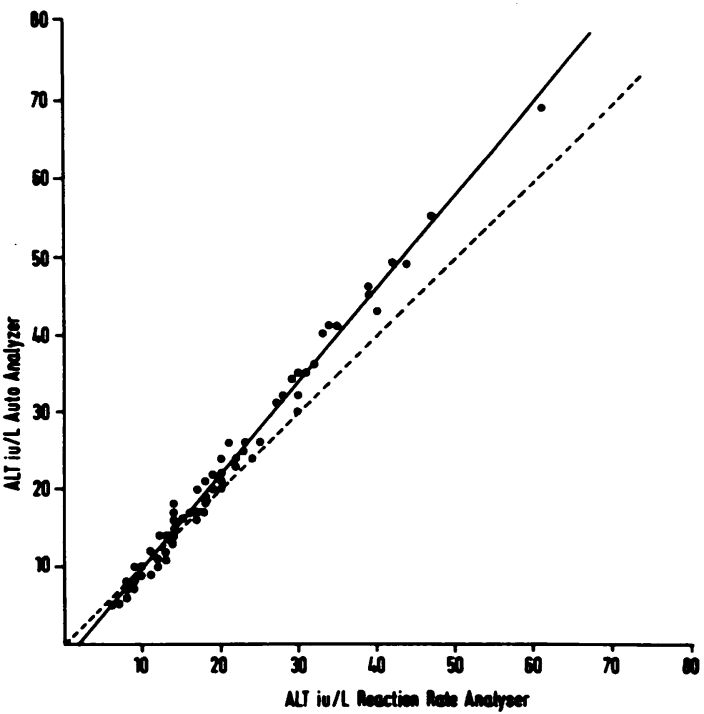

Fig 5 Correlation between reaction rate analyser and AutoAnalyzer results for patients' samples-ALT.

reaction rate analyser was inaccurately calibrated. Secondly, the slope of the line for the ALT correlations was not unity, although identical results were obtained on the two instruments for the commercial control serum used to standardize the AutoAnalyzer. This discrepancy was attributed to differences in the origin of the enzyme present in the control serum or to differences in its overall composition; similar discrepancies occurred with other control sera for both AST and ALT methods.

From these results, it seems that commercial control material is only suitable for the standardization of AutoAnalyzer transaminase methods if the numerical value to ascribe to this material is considered very carefully. Unfortunately, it is not satisfactory either to use the value given by the manufacturers or to use the value obtained in the user's laboratory using a reference method (eg, Henry et al, 1960). In our opinion, standardization should first be effected using human serum, pooled if necessary, which has been assayed by an appropriate kinetic technique. Thereafter, it may be convenient, for the day-to-day running of the method, to use commercial control material for standardization, provided that the value ascribed to this material is that previously obtained on the AutoAnalyzer using the human serum as standard.

\section{PRECISION}

One of the principal causes of lack of precision of continuous flow methods is 'carry over' (interaction). 
Experiments conducted as recommended by Broughton, Buttolph, Gowenlock, Neill, and Skentelbery (1969) with sera of high, intermediate, and low transaminase activity $(200,100$ and $20 \mathrm{iu} / 1$ for AST ; 400,150 and $25 \mathrm{iu} / 1$ for ALT) showed that carry over varied between $1.0 \%$ and $2.5 \%$.

Overall performance in terms of precision was assessed from the results for commercial control sera for within-batch precision, and from the results of serum specimens analysed on successive days for between-batch precision (Table II). There was little difference between the figures obtained for the AutoAnalyzer and the reaction rate analyser.

\section{$\cos \mathrm{TS}$}

Accurate costing of laboratory methods, considered in isolation, is almost impossible. However, an attempt has been made to obtain an approximate cost of both AutoAnalyzer and reaction rate analyser methods. The results are based on an assumed work load of 100 AST and 100 ALT analyses per day (Table III).

There is little difference in reagent costs providing these are prepared in the laboratory. Similarly, the use of the packaged AST reagents adds little to the overall cost of the analysis. However, the packaged reagent cost for the ALT analysis is unduly high, and it seems advisable to undertake the small amount of additional work involved in preparing fresh reagents in the laboratory. The cost of chemicals for the ALT analysis can be further reduced by decreasing the concentration of lactate dehydrogenase in the enzyme/coenzyme reagent and using DL-alanine (at twice the concentration) in place of L-alanine. Neither of these alterations affected the results.

Therefore, there are only insignificant differences in the overall cost of running the two types of instrument. Considerations of work load size and the

\begin{tabular}{|c|c|c|c|c|c|c|c|c|c|}
\hline & & \multicolumn{4}{|c|}{ Alanine Transaminase } & \multicolumn{4}{|c|}{ Aspartate Transaminase } \\
\hline & & $\begin{array}{l}\text { No. of } \\
\text { Samples }\end{array}$ & $\begin{array}{l}\text { Mean } \\
(i u / l)\end{array}$ & $\begin{array}{l}S D \\
(i u / l)\end{array}$ & $\begin{array}{l}C V \\
(\%)\end{array}$ & $\begin{array}{l}\text { No. of } \\
\text { Samples }\end{array}$ & $\begin{array}{l}\text { Mean } \\
(i u / l)\end{array}$ & $\begin{array}{l}S D \\
(i u / l)\end{array}$ & $\begin{array}{l}C V \\
(\%)\end{array}$ \\
\hline \multicolumn{10}{|c|}{ Within-batch precision ${ }^{1}$} \\
\hline & AA & 78 & 90 & $1 \cdot 5$ & 2 & 43 & 124 & $2 \cdot 1$ & 2 \\
\hline & RRA & 65 & 90 & $1 \cdot 7$ & 2 & 56 & 105 & $2 \cdot 5$ & 2 \\
\hline \multicolumn{10}{|c|}{ Between-batch precision ${ }^{2}$} \\
\hline $0-49$ & AA & 169 & 20 & 1.4 & 7 & 114 & 22 & $1 \cdot 0$ & 5 \\
\hline $\mathrm{iu} / 1$ & RRA & 316 & 20 & $1 \cdot 6$ & 8 & 106 & 19 & $1 \cdot 3$ & 7 \\
\hline $50-99$ & $\mathbf{A A}$ & 21 & 69 & $3 \cdot 5$ & 5 & 16 & 69 & $1 \cdot 3$ & 2 \\
\hline $\mathrm{iu} / 1$ & RRA & 43 & 66 & $2 \cdot 7$ & 4 & 40 & 65 & $2 \cdot 2$ & 3 \\
\hline $100-299$ & AA & 8 & 146 & $5 \cdot 3$ & 4 & 12 & 130 & $4 \cdot 3$ & 3 \\
\hline $\mathrm{iu} / \mathrm{l}$ & RRA & 15 & 135 & $5 \cdot 0$ & 4 & 11 & 123 & $6 \cdot 1$ & 5 \\
\hline
\end{tabular}

Table II Precision of AutoAnalyzer and reaction rate analyser

\begin{tabular}{|c|c|c|c|c|c|c|}
\hline & \multicolumn{4}{|c|}{ AutoAnalyzer } & \multicolumn{2}{|c|}{ Reaction Rate Analyser } \\
\hline & \multicolumn{2}{|l|}{$A S T$} & \multicolumn{2}{|l|}{$A L T$} & $A S T$ & $A L T$ \\
\hline & $\begin{array}{l}\text { Packaged } \\
\text { Reagents }\end{array}$ & $\begin{array}{l}\text { Freshly } \\
\text { Prepared } \\
\text { Reagents }\end{array}$ & $\begin{array}{c}\text { Packaged } \\
\text { Reagents }\end{array}$ & $\begin{array}{l}\text { Freshly } \\
\text { Prepared } \\
\text { Reagents }\end{array}$ & $\begin{array}{l}\text { Freshly } \\
\text { Prepared } \\
\text { Reagents }\end{array}$ & $\begin{array}{l}\text { Freshly } \\
\text { Prepared } \\
\text { Reagents }\end{array}$ \\
\hline Buffer/substrate & 13 & 9 & 72 & 23 & 3 & 7 \\
\hline Enzyme/coenzyme & 141 & 32 & 1200 & 139 & 36 & 40 \\
\hline Chart paper & & & & & & \\
\hline Sample cups & & & & & & \\
\hline Control/standard samples & & & & & & \\
\hline Total consumables & 269 & 156 & 1387 & $277^{2}$ & 194 & 202 \\
\hline Depreciation (6 years) & & & & & & \\
\hline Maintenance ( $5 \%$ of capital cost) & & & & & & \\
\hline Technical staff & & & & & & \\
\hline Total cost $p / 100$ tests & 729 & 616 & 1847 & 737 & 594 & 602 \\
\hline
\end{tabular}

Table III Comparative costs of AutoAnalyzer and reaction rate analyser ${ }^{1}$ 
possibility of batching of different analyses may, however, considerably alter the above figures to favour one or other instrument.

\section{RELIABILITY IN USE}

During the period of approximately two months during which the AutoAnalyzer was in use, on average three to four hours per day, the following mechanical faults occurred: (1) malfunction of sampling mechanism of sampler IV, fault rectified relatively easily; (2) dialyzer on ALT blank channel developed a leak at one end; the dialyzer unit had to be replaced. There was also a tendency for the sample probe to block; this occurred about once in every three or four runs and was easy to detect but inconvenient. A partial block which occurred in the blank side of the sample 'stream splitter' was only detected after the completion of the run when the results were seen to be too high.

Overall, the AutoAnalyzer was mechanically reliable but occasionally developed faults in the ALT flow system which were not detected immediately. In our experience, reaction rate analysers are also fairly reliable, possibly less so than AutoAnalyzers, but are prone to develop trouble in the substrate delivery pumps if these are not maintained meticulously.

\section{Conclusion}

In this study we have confirmed the original findings of Kessler, Rush, Leon, Delea, and Cupiola (1970) that the results produced by the AutoAnalyzer II for AST and ALT analysis are comparable, in terms of accuracy and precision, with those produced by reaction rate methods. Similarly, there is little difference between the overall cost of running the two types of analysis. There are, however, certain practical and theoretical considerations which may influence the user towards one or other of the two types of instrument.

Reaction rate analysis has the following advantages: (1) standardization is simple, as results may be obtained directly from the readings of absorbance change per minute. The AutoAnalyzer, on the other hand, relies on 'standards' which have been calibrated by another method. (2) Biank determination, with its associated problems, is usually unnecessary. (3) 'Carry over' is negligible.

Nevertheless, there are practical advantages that may accrue from the use of AutoAnalyzer methods.
(1) At present, the most widely used reaction rate monitor has no automatic built-in sample dilution facility, whereas the AutoAnalyzer samples automatically. (2) Many tests in the laboratory are performed as a 'group', eg, 'liver function tests' may consist of bilirubin, alkaline phosphatase, and ALT. Little additional work is involved in incorporating an AutoAnalyzer transaminase channel into such a system if the other analyses are performed on this equipment. On the other hand, considerable extra work is involved in transferring and diluting samples if different types of equipment are being used for the constituent methods in the group.

The knowledge that the AutoAnalyzer and reaction rate analyser methods of transaminase assay give similar results is also of value to those laboratories which employ multichannel AutoAnalyzer systems (eg, SMA 12/60) for much of their routine work load or for screening or profiling purposes.

Laboratories faced with the choice of whether to select continuous-flow or reaction-rate methods of transaminase assay must decide whether the theoretical and relatively minor practical analytical advantages of reaction rate analysis outweigh the greater convenience, at present, of using continuous flow methods.

If the AutoAnalyzer is used, test and blank channels must be closely matched for filters and tube sizes. Any standard used should be checked against human serum which has been itself standardized by reaction rate analysis. A serum blank may be necessary for the AST analysis if the patient is uraemic. For ALT analysis, some saving in cost can be achieved by reducing the lactate dehydrogenase concentration.

We should like to thank the Technicon Instruments Co for the loan of the AutoAnalyzer equipment.

\section{References}

Broughton, P. M. G., Buttolph, M. A., Gowenlock, A. H., Neill, D. W., and Skentelbery, R. G. (1969). Recommended scheme for the evaluation of instruments for automatic analysis in the clinical biochemistry laboratory. J. clin. Path., 22, 278-284.

Henry, R. J., Chiamori, N., Golub, O. J., and Berkman, S. (1960). Revised spectrophotometric methods for the determination of glutamic-oxalacetic transaminase, glutamic-pyruvic transaminase and lactic acid dehydrogenase. Amer. J. clin. Path., 34, 381-398.

Kessler, G., Rush, R. L., Leon, L., Delea, A., and Cupiola, R. (1970). Automated $340 \mathrm{~nm}$ measurement of SGOT, SGPT and LDH. Clin. Chem., 16, 530-531.

Smith, A. F., Brown, S. S., and Taylor, R. (1970). Assessment of an automatic enzyme reaction rate monitor. Clin. chim. Acta, 30, $105-113$. 